\title{
Correct identification of a myocardial perfusion SPECT lesion leads to early diagnosis of lung cancer
}

\author{
Suresh Viswanathan, FRACP, ${ }^{\mathrm{a}}$ Jan Mueller-Brand, $M D,{ }^{a}$ Matthias E. Pfister, $M D$, \\ FACC, ${ }^{\mathrm{b}}$ and Michael J. Zellweger, $\mathrm{MD}^{\mathrm{b}}$
}

Case presentation. A 62-year-old man presented with recent onset of typical angina. Dual-isotope myocardial perfusion scintigraphy (MPS) was performed. The rest and stress images are shown in Figure 1. The raw data image of the thorax is shown in Figure 2.

The findings were consistent with an inferior wall scar with a small amount of peri-infarct ischemia; however, the anterior wall was difficult to interpret because of the prominent overlying extracardiac activity. This activity can be seen in the anterior projection image of Figure 2. The single photon emission computed tomography-reconstructed images of Figure 1 confirm that the extraneous activity is within the chest cavity and located above the left ventricle.

From the Departments of Nuclear Medicine ${ }^{\mathrm{a}}$ and Cardiology, ${ }^{\mathrm{b}}$ University Hospital, Basel, Switzerland

Reprint requests: Michael Zellweger, MD, Department of Cardiology, University Hospital, Petersgraben 4, CH-4031 Basel, Switzerland; mzellweger@uhbs.ch.

J Nucl Cardiol 2004;11:361-3.

$1071-3581 / \$ 30.00$

Copyright (C) 2004 by the American Society of Nuclear Cardiology. doi:10.1016/j.nuclcard.2004.03.003
This abnormal activity was present in the thallium 201 and technetium $99 \mathrm{~m}$ sestamibi raw data. Because of the high likelihood of malignancy, a chest computed tomogram was ordered (Figure 3). Bronchoscopy followed, from which the lesion was diagnosed as nonsmall cell carcinoma, and in addition, the patient underwent positron emission tomography for staging purposes (Figure 4). On the basis of the positron emission tomography result, the patient was staged as $\mathrm{N} 2 \mathrm{M} 0$. The relatively early diagnosis allowed the patient to proceed to surgery with curative intent.

Discussion. Noncardiac lesions are present in about $1.7 \%$ of MPS studies. ${ }^{1}$ This illustrates the importance of examining all of the information provided by MPS, as Tl-201 and Tc-99m sestamibi are taken up not only by the myocardium but also by a wide variety of benign and malignant lesions. ${ }^{2}$

\section{Acknowledgment}

The authors have indicated they have no financial conflicts of interest.

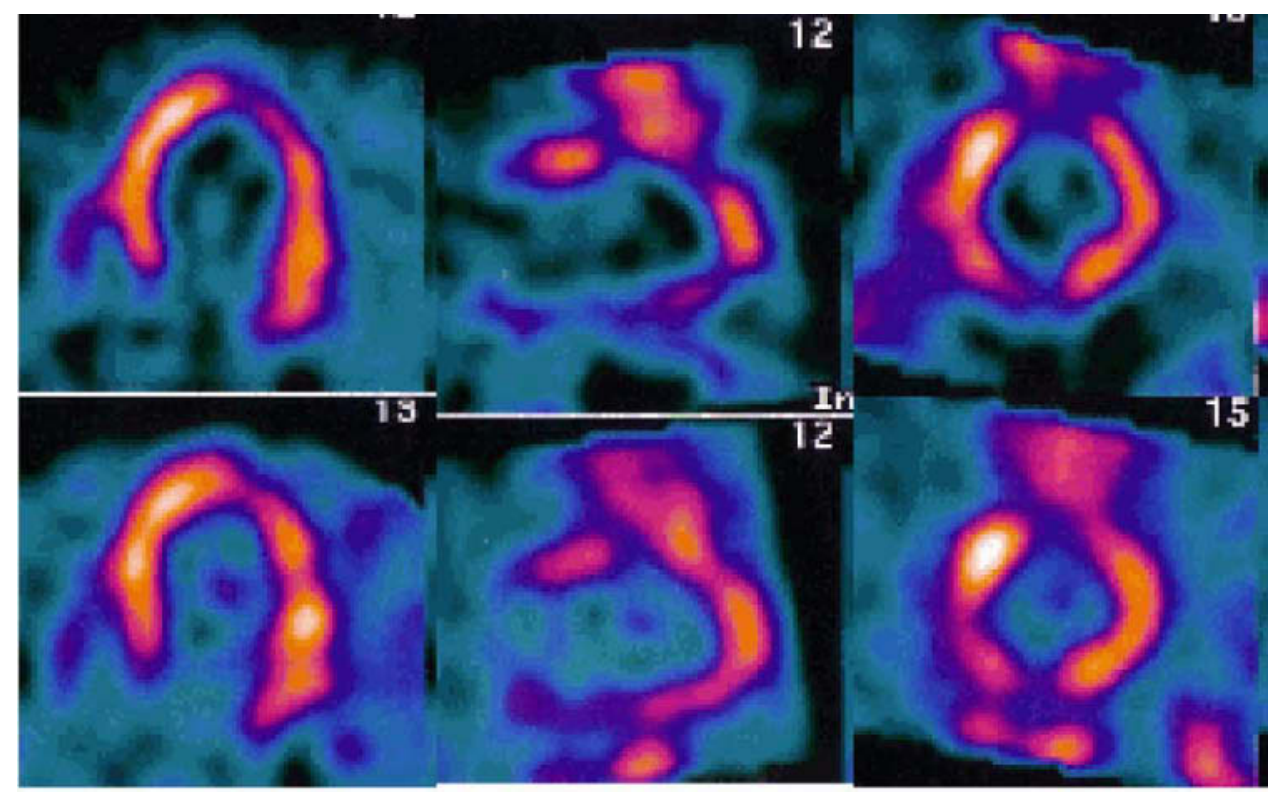

Figure 1. Horizontal long-axis (left), vertical long-axis (middle), and short-axis (right) images of the left ventricle, with stress images (Tc-99m sestamibi) shown in the top row, and the corresponding rest images (Tl-201) shown in the bottom row. 


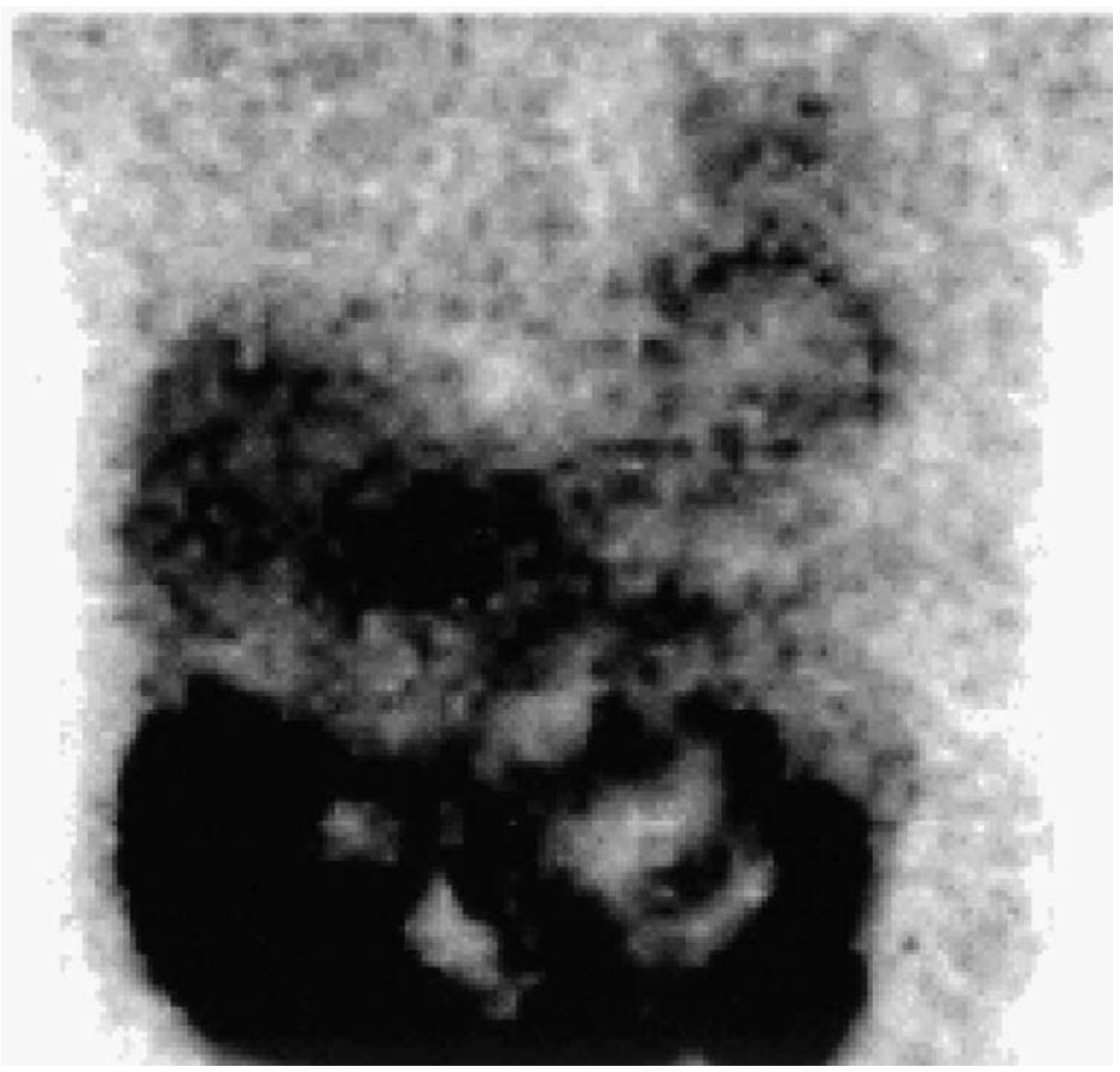

Figure 2. Raw data view of the thorax (Tc-99m sestamibi).

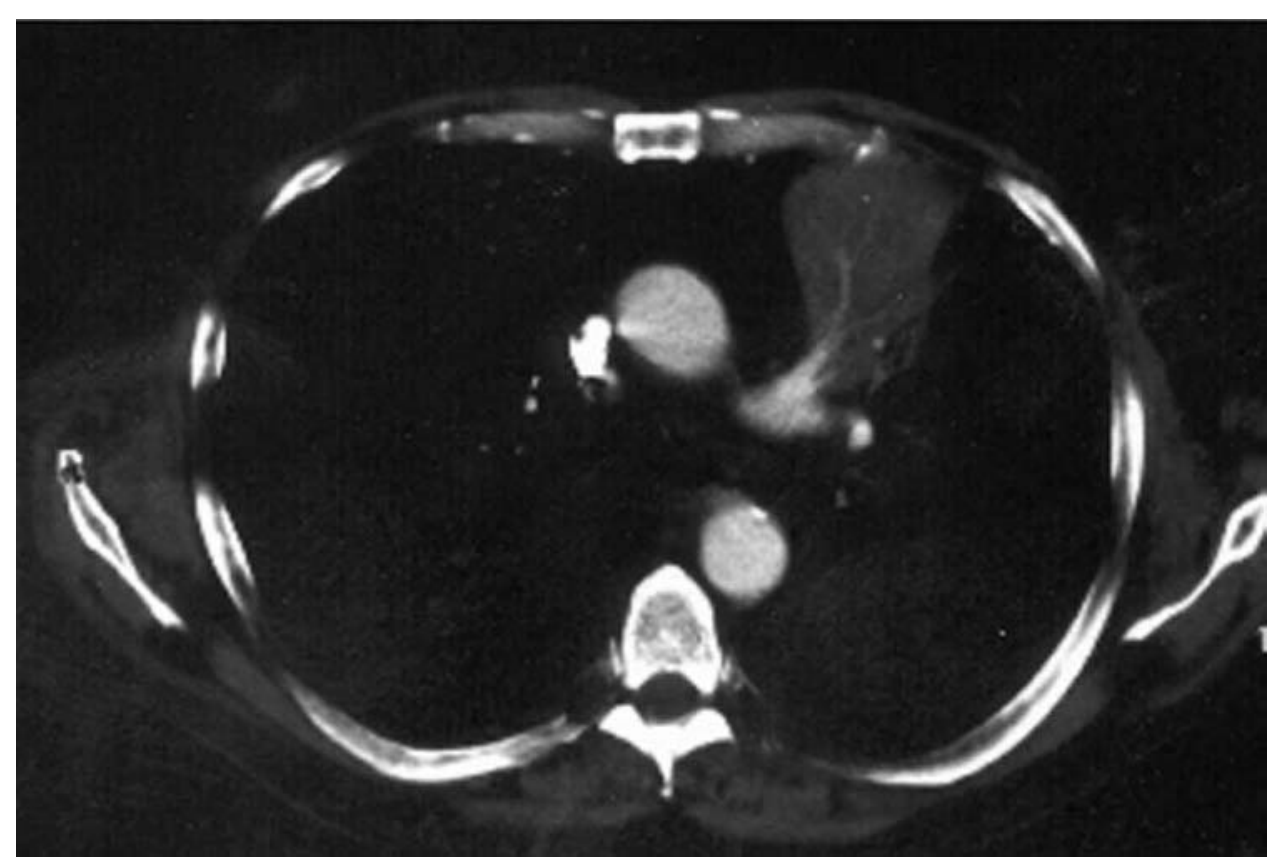

Figure 3. Chest computed tomogram shows a mass in the left upper lobe with enlarged pericarinal lymph nodes and complete atelectasis of the left upper lobe and part of the lingula. 

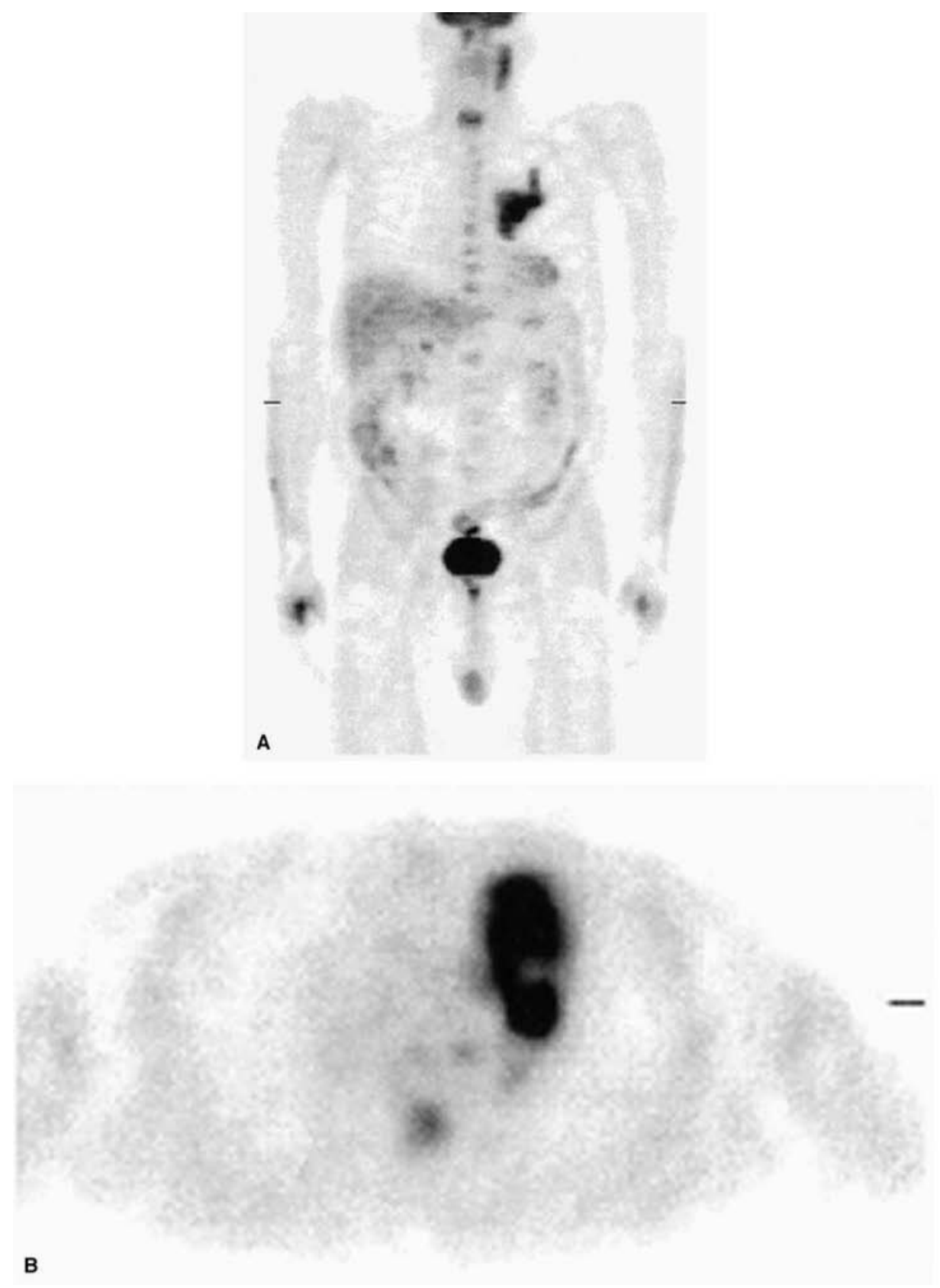

Figure 4. Positron emission tomography study, with fluoro-2-deoxy-D-glucose (F-18 FDG) used as the tracer, shown in the whole-body projection view (A) and transaxial view (B), demonstrates intense tumor uptake in the left upper lobe, near the left hilum, with lower-grade uptake in the surrounding atelectasis. F-18 FDG uptake outside the lung in the whole-body view is physiologic with no evidence of distant metastases.

\section{References}

1. Williams KA, Hill KA, Sheridan CM, et al. Noncardiac findings on dual-isotope myocardial perfusion SPECT. J Nucl Cardiol 2003;10: 395-402.

2. Sutter CW, Joshi MJ, Stadalnik RC. Noncardiac uptake of technetium-99m MIBI. Semin Nucl Med 1994;24:84-6. 